Instituto Internacional de Investigación y Desarrollo Tecnológico Educativo INDTEC, C.A.

DOI: https://doi.org/10.29394/Scientific.issn.2542-2987.2018.3.7.12.231-249

OAI-PMH: http://www.indteca.com/ojs/index.php/Revista Scientific/oai

\title{
Jardines Ornamentales como Estrategia de Participación local en el Embellecimiento de Áreas Verdes
}

\author{
Autora: Omaira Andreina Márquez Ramírez \\ Universidad Pedagógica Experimental Libertador, UPEL \\ omairamarquez89@gmail.com \\ Barinas, Venezuela
}

\section{Resumen}

La participación local es considerada, como mecanismo de acción para el rescate de las áreas verdes, por tanto la investigación persigue, crear una conciencia conservacionista en los habitantes que hacen vida en la comunidad, en cuanto a la necesidad de mantener un ambiente en buenas condiciones, por consiguiente, plantea como meta fundamental promover la construcción de jardines ornamentales como estrategia de participación local en el embellecimiento de áreas verdes en la Comunidad Costa de Morrocoy, Municipio Barinas, Estado Barinas. Para los aportes teóricos que sustentan la investigación se consultaron, diferentes autores y enfoque relativos a la participación local y jardines ornamentales. La misma se desarrolló dentro del paradigma cualitativo y centrado en una investigación acción participante, en relación al diseño es de campo, por cuanto la recolección de datos se realizó directamente de los sujetos investigados, en lo que respecta a los informantes, se tomaron, cuatros (04) sujetos objetos de estudio, en cuanto a la técnica e instrumento de recolección de datos se utilizó la entrevista así como el guion de entrevista cuyos resultados se analizarán mediante el proceso de triangulación la misma con la intencionalidad de dar repuesta a los propósitos de la investigación.

Palabras clave: participación comunitaria; comunidad; medio
ambiente. 


\title{
Ornamental Gardens as a Strategy for Local Participation in the Green Area Embellishment
}

\begin{abstract}
Local participation is considered, as a mechanism of action for the rescue of green areas, so research seeks to create a conservationist conscience in the inhabitants that make life in the community, as to the need to maintain an environment in good condition, Therefore, aims to promote the construction of ornamental gardens as a strategy for local participation in the beautification of green areas in the Community of Morrocoy Coast, Barinas Municipality, Barinas State. For the theoretical contributions that support the research were consulted, different authors and approach related to local participation and ornamental gardens. It was developed within the qualitative paradigm and focused on a participatory action research, in relation to the field design, since the data collection was done directly from the subjects investigated, with respect to the informants, were taken, four (04) subjects of study, as for the technique and instrument of data collection was used the interview as well as the interview script whose results will be analyzed through the triangulation process itself with the intention of giving a response to the purposes of the investigation.
\end{abstract}

Keywords: community participation; communities; environment. 


\section{Introducción}

En el ámbito mundial, los jardines ornamentales son llevados a la práctica como estrategias ecológicas, para el embellecimiento de las áreas verdes, considerándose en respuesta alternativas para minimizar el impacto ambiental que fomenta la actividad humana tradicional, especialmente sobre el suelo y el agua. Consiste en una serie de medidas con el fin de obtener un mejor espacio para la práctica del esparcimiento y la recreación, a la par de preservar el ambiente. Estas alternativas contemplan el uso propicio de la tierra, tomando en cuenta su potencialidad para cada tipo de plantas en particular.

Al respecto Loyo (2013), considera que la "aplicación de métodos ecológicos disminuye la degradación de los suelos, evadiendo así la acción de los agentes erosivos, por consiguiente, la siembra de plantas ornamentales de manera tradicional, fortalecen los aspectos ecológicos, por cuanto permite crear conciencia conservacionista en quienes practican de este estilo de cultivo". (pág. 4). De igual manera, se busca mejorar el uso de los fertilizantes y el manejo adecuado del agua, bien sea de lluvia o la utilizada para riego; y se procura el uso de abonos verdes como cultura ecológica, con el fin de darle un mayor aprovechamiento a los recursos naturales, así como la conservación de los mismos.

Bajo esta tendencia, Sierra (2012), señala "con la puesta en práctica de medidas ambientales para la preservación de áreas verdes se han ido creando modelos de desarrollo independientes, que aseguraran espacios naturales activos en las comunidades y sus adyacencias" (pág. 98). Esto, ha impulsado una serie de valores y actitudes necesarias para un cambio hacia comportamientos más respetuosos con el medio ambiente, se obtendrá un ambiente equilibrado, se aprovechará la diversificación y protección de las plantas, conservación de aguas, suelos, y otros. 
Respecto, a las áreas verdes Ojeda y Espejel (2014), señalan:

Con una visión más amplia las áreas verdes están constituidas por todos aquellos parques, jardines, camellones, glorietas, áreas naturales y deportivas que forman parte de determinada ciudad, las cuales han pasado de ser elementos secundarios del paisaje, con fines solamente estéticos y recreativos, a convertirse en áreas de gran importancia, debido su funcionabilidad y a los beneficios ecológicos que aportan a la sociedad. (pág. 5).

En consideración a ello se estima, que con la práctica de la construcción de los jardines ornamentales se permitirá desarrollar la sensibilización ante distintas problemáticas ambientales presentes, en las comunidades, de esta manera adopten una conciencia sobre la incidencia de las actividades que se realizan sobre el equilibrio del medio, dándole la importancia de preservar la biodiversidad, y de actuar de manera responsable y respetuosa con respecto a la conservación del ambiente, lo que afirma la misma autora, Kreuter (2005), en relación a los jardines.

Los define como una expresión en la que recurre a diferentes elementos, plantas y medios técnicos para la creación de espacios verdes determinado, un jardín no solo se cuidara con esmero, la salud del suelo, se le abonara reciclando sus propios materiales, por tanto, para lograr un buen jardín es necesario saber distribuirlas de acuerdo con sus especies. (pág. 11).

Así mismo, señala Menes (2006); “...en los actuales momentos se han consolidado y profundizado las democracias, los procesos de descentralización y las redefiniciones del papel del Estado en materia de formulación de políticas e instrumentación de programas sociales" (pág. 18). En este sentido, la noción del país y del gobierno local incluye nuevas formas para establecer la participación. En muchos casos llega a la táctica de la relación con las organizaciones comunitarias, ajustada a la corresponsabilidad de quienes conforman dichas organizaciones.

También, la participación en el desarrollo ambiental de cualquier 
organización social, está presta a abordar las diferentes situaciones que de manera directa generen necesidades que desde los entes gubernamentales no puedan ser resueltos. Al respecto, Villegas (2014), señala que en las comunidades "... deben existir planes y proyectos enmarcados en el desarrollo local como una forma de garantizar el curso inminentemente eficaz y efectivo de las organizaciones comunitarias en función al desarrollo social, político, económico, ambiental anhelado" (pág. 14). Es por ello, que desde este punto de vista, la participación ciudadana como hecho político y social se vislumbra en los alcances que de manera holística, ésta debe proyectar para lograr las metas que desde este proceso se planifican.

En lo que respecta a Latinoamérica, Restrepo (2015), considera que, los jardines ornamentales, es una medida que viene dando respuesta a ciertas políticas de embellecimiento natural; la cual se puede desarrollar en terrenos pequeños existentes en la comunidad y sus casas, donde se planten especies ornamentales que embellezcan el espacio comunitario. A su vez, se puede reflexionar sobre la necesidad de acometer acciones que conlleven a una integración comunidad institución educativa para una mejor conservación del ambiente y calidad de vida comunitaria.

En el caso de Colombia Medellín, la jardinería se va articulando cada vez más a las tendencias actuales del paisajismo contemporáneo, caracterizado por el eclecticismo o fusión de estilos y conceptos de jardín; y asistido en gran medida por la tecnología como rasgo clave del diseño y la función. Una concurrencia potencialmente innovadora que sin embargo, suele desembocar en prácticas paisajísticas o jardineras aligeradas o prestadas, gracias a la inmediatez electrónica de la información y la rápida divulgación y exportación de especies, estilos y diseños que se llevan a ciudades de muy distintas latitudes, implantando especies, técnicas y estilos sin mayor dependencia del contexto ecológico local.

En consideración a Venezuela, Díaz (2014), expresa que, en Venezuela 
existe una gran diversidad de plantas con importantes cualidades ornamentales que son ignoradas por los arboricultores y han sido poco estudiadas desde el punto de vista urbanístico. La divulgación de los beneficios y usos de estas plantas constituye un aspecto fundamental a considerar en la planificación y la expansión de las comunidades urbanas y rurales, visto que en los últimos años se ha ido promoviendo la concepción integral del ambiente, a través de actividades socio ambientales, la protección de la biodiversidad y socio diversidad; así como comprender y abordar la problemática ambiental, integrando a todos los actores comunitarios, educativos y gubernamentales.

De acuerdo con todo lo ante planteado, surge la participación protagónica de las familias y comunidades, desde la visión de la autogestión, la corresponsabilidad y el desarrollo integral. Estas vienen a llenar ese espacio que anteriormente era dispuesto para la circulación u ocio, que puede ir desde una ventana, pequeños materos, hasta jardines familiares y comunales, ahora aprovechados para el embellecimiento comunitario, dirigidos fundamentalmente a garantizar ambientes naturales abiertos y saludables, explotando las bondades que brinda el rico entorno tropical.

Cabe destacar que a través de los jardines ornamentales comunitarios, se busca fortalecer la cultura del trabajo solidario comunitario, de igual manera la importancia del cuidado de las plantas, la adquisición de valores que permitan desarrollar una cultura ambiental, para el equilibrio de la naturaleza, así como los mecanismo para el buen uso que se le debe dar a los recursos naturales, de esta manera los jardines ornamentales comunitarios son parte fundamental, para la recuperación de áreas verdes, visto que a pesar de las políticas ambientales con las que goza el país, se evidencia con profunda preocupación el deterioro de las áreas verdes.

Bajo esta perspectiva y ante la realidad de los problemas ambientales que viene presentando el Estado Venezolano, tomando como base el deterioro 
de la capa vegetal producto del proceso de construcción y ampliación de zonas pobladas, se toma como objeto de estudio la Comunidad Costa de Morrocoy, Parroquia San Silvestre, Municipio Barinas, Estado Barinas, por considerar necesario, puesto que dicha comunidad carece de jardines ornamentales, contando con espacios suficientes para construcción de los mismos, en tal sentido el rescate de áreas verde, se debe a la falta de motivación y concientización de los habitantes de la comunidad, en tal sentido se pretende con el abordaje de la investigación la promoción de construcción de jardines de jardines ornamentales con la participación de la comunidad, considerando que de esta manera se fortalecerá los valores ciudadanos, en la búsqueda de la conservación del ambiente.

Por tanto, el propósito general de la investigación se fundamenta en Promover la construcción de jardines ornamentales como estrategia de participación local en el embellecimiento de áreas verdes en la Comunidad costa de Morrocoy, Parroquia San Silvestre, Municipio Barinas, Estado Barinas.

\section{Referentes Teóricos}

\subsection{Desarrollo Local}

Morales (2006), consideró que las iniciativas de desarrollo local toman fuerza en la década de los años ochenta del siglo pasado, con el agotamiento del modelo fondista de desarrollo que, para esa época, no se ajustaba a las exigencias del desarrollo alcanzado por las fuerzas productivas. El progreso tecnológico de esa etapa planteaba la necesidad de buscar formas productivas mucho más flexibles y eficientes que garantizaran mayor calidad de las producciones, a tenor de las nuevas exigencias de la demanda mundial.

Según la teoría del desarrollo económico local, resultaba extremadamente difícil lograr lo antes expuesto en los marcos del modelo de producción forista. Comienza a tomar fuerza la idea de que esa 
reestructuración tecnológica y organizativa, ese ajuste productivo mucho más flexible, es de hecho más factible en el ámbito del territorio local, el potenciar los recursos de carácter endógeno. En tal sentido se produce un cambio importante en la conceptualización del desarrollo junto a la visión del desarrollo exógeno que promueve la atracción de capitales y empresas externas para impulsar el crecimiento económico de un territorio.

De esta manera destacó la fuente, que toma fuerza, así, la noción del desarrollo económico local, donde los procesos de desarrollo local se producen gracias a la utilización eficiente del potencial económico local, que se ve facilitado por el funcionamiento adecuado de las instituciones y mecanismos de regulación del territorio.

\subsection{Jardines ornamentales}

De la Cruz (2011), Señaló que "un jardín es un espacio abierto al aire libre poblado de especies vegetales de diversas características ornamentales y con algunas construcciones para el descanso y el recreo de los humanos." (pág. 5). Es por tanto un espacio creado por y para los hombres sin mayor pretensión que la del disfrute y contemplación humanos. Son espacios que se hacen sin una finalidad económica ya que por sí mismos no reportan beneficio alguno, pero sí tenemos que tener en cuenta que es necesario dedicar tiempo y dinero para su correcto mantenimiento, ya que si dejamos que muchas de esas especies crezcan y se desarrollen según su porte natural se perderían las características ornamentales del diseño original del jardín, y muchas de ellas no cabrían con los marcos de plantación que se utilizan en jardinería. Es necesario, por tanto, tener en cuenta que habrá que destinar una partida económica al mantenimiento del jardín.

De igual manera expresó la fuente que un jardín ornamental, sólo se puede conservar si ha sido bien plantado, para lo cual es imprescindible conocer y estudiar la jardinería, todo lo relacionado con las plantas, el terreno 
y el riego, que dependerá de la necesidad hídrica de allí que un jardín alcanza la plenitud de su belleza después de muchos años de haber sido plantado; su construcción dura por término medio dos años y su permanencia refleja las condiciones en que fue establecido. El jardín es un lujo, un lugar de recreo y ocio, en el trazado del jardín hay que tener en cuenta la orientación, el clima, el agua, el suelo, la superficie total y la orografía, la vegetación existente, si la hubiera, y cómo se quiere o gustaría que fuera. La orientación del terreno es decisiva para proyectar un jardín y hacer la selección de las plantas que son más adecuadas para cada zona. Finalmente expresó que en los jardines ya plantados no hay orientaciones precisas porque los cerramientos, árboles y el resto de vegetación plantada han modificado en su interior las horas de sol, creando sus propias sombras y microclimas, en tal sentido plantar jardines en áreas comunitarias son de gran ventaja para el recate de áreas verdes ociosas las cuales al ser utilizadas se convierten en beneficio de la comunidad, por ser parte en las horas de ocio y esparcimiento.

\subsection{Participación comunitaria}

Bajo la concepción de participación comunitaria se expresa, que las comunidades organizadas en la búsqueda del bien común y la solución de las problemáticas que puedan surgir, pueden adoptar mecanismos de participación, este sentido, el marco legal de la constitución de la República Bolivariana de Venezuela hace alusión a la participación ciudadana, en tal caso el artículo 62 considera, textualmente lo siguiente: Todos los ciudadanos y ciudadanas tienen el derecho de participar libremente en los asuntos públicos, directamente o por medio de sus representantes elegidos o elegidas. La participación del pueblo en la formación, ejecución y control de la gestión pública es el medio necesario para lograr el protagonismo que garantice su completo desarrollo, tanto individual como colectivo.

De esta manera, la participación consciente y voluntaria de los 
ciudadanos en la solución de problemáticas socioambientales, es factor de vital importancia, que se debe tomar en consideración para la promoción de actividades comunitarias que permitan insertar la participación de los ciudadanos, así como la formación de valores basados en la conservación del ambiente, en tal caso la construcción de jardines ornamentales en beneficio de la ciudadanía.

\section{Bases Legales}

En lo que concierne a las bases legales la presente investigación tendrá apoyo desde un punto de vista legal en, la Constitución de la República Bolivariana de Venezuela, donde se refleja lo concerniente con la preservación del medio ambiente.

En tal sentido el artículo 127. Expresa lo siguiente:

Es un derecho y un deber de cada generación proteger y mantener el ambiente en beneficio de sí misma y del mundo futuro. Toda persona tiene derecho individual y colectivamente a disfrutar de una vida y de un ambiente seguro, sano y ecológicamente equilibrado.

En lo que respecta a este artículo, nuestra constitución tiene un rol protagónico dentro de la propuesta de investigación, considerando que es el elemento jurídico que apoya esta investigación, en virtud que destaca el deber que tiene la ciudadanía en proteger el ambiente.

De igual manera destaca la carta magna en sus artículos (128 y 129) El deber que tiene el estado de desarrollar políticas ambientales, que permitan atender la realidad tanto ecológica como ambiental de las poblaciones, en la búsqueda de conservar el ambiente. 


\subsection{Ley Orgánica del Ambiente. Título III de la planificación del ambiente}

(2006).

En función a ello, el artículo 26 de la Ley Orgánica del Ambiente, contempla lo siguiente:

La planificación del ambiente está circunscrita a un sistema integral y jerarquizado de planes, cuyo instrumento fundamental el plan nacional de ordenación del territorio. El plan nacional del ambiente se desarrollará con carácter vinculante por todos los órganos y entes del poder público nacional, estatal y municipal, así como los consejos comunales. Los planes ambientales, nacionales, regionales, estadales, municipales y locales conforman el sistema nacional para la planificación del ambiente y son instrumentos fundamentales de la gestión pública en materia ambiental.

De acuerdo con lo previsto en este artículo, se hace referencia a los objetivos que se deben tomar en consideración para el proceso eficaz de la planificación en materia del ambiente, el cual es de vital importancia para el desarrollo de actividades relativas, a la conservación del ambiente, de allí que estos artículos son relevantes en la investigación abordada, siendo que permiten consolidar el contexto de la planificación.

\subsection{Ley Orgánica de Seguridad de la Nación Título I. Disposiciones fundamentales.}

Artículo 1. La presente Ley tiene por objeto regular la actividad del Estado y la sociedad, en materia de seguridad y defensa integral, en concordancia a los lineamientos, principios y fines constitucionales.

Artículo 2: Seguridad de la Nación. La seguridad de la Nación está fundamentada en el desarrollo integral, y es la condición, estado o situación que garantiza el goce y ejercicio de los derechos y garantías en los ámbitos económico, social, político, cultural, geográfico, ambiental y militar de los principios y valores constitucionales por la población, las instituciones y cada 
una de las personas que conforman el Estado y la sociedad, con proyección generacional, dentro de un sistema democrático, participativo y protagónico, libre de amenazas a su sobrevivencia, su soberanía y a la integridad de su territorio y demás espacios geográficos.

De acuerdo a estos artículos, se considera el deber que tiene el estado en velar por el desarrollo de políticas ambientales, que permitan el fortalecimiento de la participación activa y protagónica de la sociedad, de allí, la importancia tanto a nivel jurídico como social y político que se le ha dado a la educación ambiental, mediante una serie acciones conducentes al manejo integral del sistema ambiental, con el objetivo de lograr una adecuada calidad de vida, previniendo o mitigando los problemas ambientales, buscando la manera más viable de conseguir un equilibrio para el uso racional de los recursos , protección y conservación del ambiente.

\section{Marco Metodológico}

\subsection{Naturaleza de la Investigación}

El presente estudio, se cimienta en promover la construcción de jardines ornamentales como estrategia de participación local en el embellecimiento de áreas verdes en la Comunidad costa de Morrocoy, Parroquia San Silvestre, Municipio Barinas, Estado Barinas. El estudio está sustentado en el enfoque cualitativo, dado que trata de comprender a las personas dentro del marco de referencia de ellas mismas, de manera que es esencial experimentar la realidad tal como otros la experimentan. Según Martínez (2008), afirma que "la investigación cualitativa trata de identificar la naturaleza profunda de las realidades, su estructura dinámica. Lo que se desea es el análisis de forma detallada de un asunto o actividad particular, es decir; lo que se busca es solucionar de forma detallada la situación o problemática estudiada" (pág. 24). 


\subsection{Tipo de Investigación}

Con respecto al tipo de investigación se orientará mediante la Investigación Acción participativa, esto significa que el investigador participa también en el estudio y se encuentra dentro del contexto observado el fenómeno, además comparte con los habitantes de la comunidad. Al respecto Hernández, Fernández, y Baptista (2008), señalan que:

Los estudios descriptivos miden de manera independiente los conceptos o variables con los que tiene que ver; aunque desde luego puede integrar las mediciones de cada una de dichas variables para decir como es y se manifiesta el fenómeno de interés. Puesto que se trabaja en un área geográfica y con "personas" o "sujetos", quienes son las fuentes de conocimientos, utilizando como instrumentos diferentes tipos de observación, encuestas, entrevistas, cuestionarios, test o pruebas para hacer indagaciones (pág. 16).

De acuerdo con lo referido anteriormente se hace pertinente la presente investigación, puesto que el propósito consiste en la intervención de la investigadora para promover la construcción de jardines ornamentales como estrategia de participación local en el embellecimiento de áreas verdes en la Comunidad costa de Morrocoy, Parroquia San Silvestre, Municipio Barinas, Estado Barinas.

\subsection{Diseño de la Investigación}

El estudio quedo Afianzado dentro de un diseño de campo. En tal sentido Según el autor Arias (2007), define: La investigación de campo es aquella que consiste en la recolección de datos directamente de los sujetos investigados, o de la realidad donde ocurren los hechos (datos primarios), sin manipular o controlar variable alguna, es decir, el investigador obtiene la información, pero no altera las condiciones existentes. De allí su carácter de investigación no experimental.

Es importante reflejar que, por medio de la instrumentación del diseño 
establecido, se considera oportuno llevar a cabo un diagnóstico de la realidad que tiene que ver construcción de jardines ornamentales como estrategia de participación local en el embellecimiento de áreas verdes en la Comunidad costa de Morrocoy, Parroquia San Silvestre, Municipio Barinas, Estado Barinas.

\subsection{Etapas del Estudio.}

a). La Observación Participante: Esta se desarrollará mediante diferentes visitas realizadas a la comunidad al objeto de ir percibiendo la problemática según las opiniones y manifestaciones de los miembros de la comunidad, lo cual permitirá identificar el problema de manera puntual como el apoyo de los miembros de la comunidad.

b). La Investigación Participativa: Una vez identificado el problema, se procederá a describir os aspectos teóricos-metodológicos que hacen referencia al tema, razón por la cual se analizarán diferentes estamentos conceptuales y legales, que sostienen desde el punto de vista teórico la investigación, De igual forma se describirán los rasgos metodológicos en los que se inscribe el estudio, estos orientan el estudio metodológicamente de manera significativa.

c). La Acción Participativa: Esta fase se desarrollará mediante la interacción informativa y comunicativa entre los investigadores y la comunidad, dando paso a la propuesta de acciones que persiguen dar respuesta inmediata al problema evidenciado.

d). La evaluación: En esta última fase, se aplicarán los medios de verificación que permitirán establecer los logros, debilidades y situaciones por mejorar durante la puesta en marcha del plan acción elaborado para dar respuesta al problema que se ha planteado. 


\subsection{Informantes Claves}

Un informante clave es aquella persona que cuenta con el conocimiento suficiente del área investigada, y su aporte y experiencia resulta valiosa para el tema en estudio, con relación a ello, Parra (2006), señala que, la unidad de observación o informante, es aquella por medio de la cual se obtiene la información, es decir, es la unidad informante.

Por lo expuesto, se indica que las unidades informantes del actual estudio fueron cuatro (04) individuos que hacen vida en la Comunidad de Morrocoy Abajo, pertenecientes a la parroquia San Silvestre, los cuales fueron escogidos por ser miembros activos de la comunidad, conocedores de las situaciones que afectan el entorno comunitario.

\subsection{Técnicas de investigación y recolección de datos}

Respecto a las técnicas de recolección de datos, estas pueden considerarse como los procedimientos que manipula el investigador para recolectar la información requerida en el diseño de la investigación. A lo expuesto anteriormente Arias (2012), expresa "son las distintas formas o maneras de obtener la información" (pág. 53). Dada la naturaleza de esta investigación, y en función de los datos que se requerían, las técnicas que se utilizaron fueron la entrevista y la observación, las cuales se aplicaron a los participantes de la investigación abordada en la comunidad, en tal caso se tomaron para cuatro (04) individuos de los cuales se intenta obtener información relevante al objeto de la investigación abordada.

En cuanto a los instrumentos de registros de las entrevistas, se utilizaron grabadora y hojas de recordatorio. Todo ello con la intención de dar fidelidad a las respuestas y opiniones para una mejor utilización de los datos.

\section{Conclusiones}

En lo relativo a las consideraciones finales de la investigación surgen 
las conclusiones, tomando como base los objetivos pertinentes al estudio.

De esta manera el primer objetivo definido diagnosticar las condiciones de los jardines que se encuentran en la Comunidad costa Morrocoy, Parroquia San Silvestre, Municipio Barinas para la recuperación de espacios de recreación y esparcimiento para ello, se realiza la descripción detallada del contexto que se desea mejorar, es describir lo más objetivamente posible la realidad y de allí determinar si se hace necesario presentar una alternativa para regenerar la problemática.

En cuanto al segundo objetivo, el cual hace referencia a Planificar estrategias de participación local orientadas a la construcción de jardines comunitarios para el embellecimiento de áreas verdes en la Comunidad costa de Morrocoy, Parroquia San Silvestre, Municipio Barinas, se especifica en los aportes pedagógicos que se les brinde que se le puede brindar a la comunidad para mejorar la problemática.

En lo que respecta al tercer objetivo, ejecutar mediante la participación de los miembros de la localidad el embellecimiento de las áreas verdes de la Comunidad Costa de Morrocoy, Parroquia San Silvestre, Municipio Barinas, se busca con ello, consolidar la propuesta de involucrar a la comunidad en las actividades relativas a la conservación del ambiente.

Finalmente, el cuarto objetivo, Evaluar la participación local evidenciada durante la construcción de jardines comunitarios para el embellecimiento de áreas verdes en la Comunidad

Este objetivo de igual manera persigue, crear una conciencia conservacionista en los habitantes que hacen vida en la comunidad. Para que tomen conciencia en cuanto a la necesidad de mantener un ambiente en buenas condiciones, a través de la participación ciudadana

\section{Referencias}

Arias, F. (2012). El Proyecto de Investigación. Introducción a la 
Metodología Científica. 6ta edición, Venezuela: Espíteme.

Arias, F. (2007). El Proyecto de Investigación, introducción a la metodología científica. 5ta. edición. Caracas, Venezuela: Episteme, C.A.,

Constitución de la República Bolivariana de Venezuela (2000). Gaceta Oficial

Extraordinaria de la República Venezuela no 5453, del 24 de marzo. Caracas, Venezuela.

De la Cruz, R. (2011). Iniciación a la jardinería. España: Aula Mentor.

Díaz, J. (2014). Arbustos Ornamentales de Maracaibo, Estado Zulia, Venezuela Ornamental. Rev. Fac. Agron. (LUZ). 2014, Supl. 1: 224.

Hernández. S, Fernández, C. \& Baptista, P. (2008). Metodología de la Investigación. 3r Edición. México. Editorial: McGraw-Hill.

Kreuter, M. (2005). Jardín y huerto biológicos. España: Editorial: MundiPrensa.

Ley Orgánica del Ambiente (2006). Gaceta Oficial de la República Venezuela no 5833, del 22 de diciembre. Caracas, Venezuela.

Loyo, Q. (2013). Aprendamos Horticultura. Plantas Ornamentales. [Artículo en línea]. Recuperado de:

http://aprendamoshorticulturaenlaeta.blogspot.com/2015/06/plantasornamentales.html

Martínez, M. (2008). Ciencia y arte en la metodología Cualitativa. México: Trillas.

Menes, F. (2006). Visión Ambiental de los Recursos Naturales en la actualidad. Caracas, Venezuela: Fundación Natural Editores.

Morales, M. (2006). Desarrollo local sostenible. [Artículo en línea]. Recuperado de: http://www.Redalyc.org/pdf/4255/425541310004.pdf

Ojeda, L. \& Espejel, I. (2014). Cuando las áreas verdes se transforman en paisajes urbanos. La visión de Baja California. México

Parra, J. (2006). Guía de Muestreo. Primera Edición. Dirección de Cultura, 
Venezuela: Universidad del Zulia.

Restrepo, L. (2015). Jardines Ornamentales Urbanos Contemporáneos: Transnacionalización, Paisajismo y Biodiversidad. Un Estudio Exploratorio en Medellín, Colombia. [Artículo en línea]. Recuperado de: http://www.scielo.org.co/pdf/rfnam/v68n1/v68n1a16.pdf

Sierra, M. (2012). Recursos Ambientales y Políticas de Gestión. Ediciones Bellas Monte. Caracas, Venezuela: Universidad Central de Venezuela (UCV).

Villegas, C. (2014). Análisis de los Ambientes Naturales en Latinoamérica. Revista Ambientalista de Publicación Semestral. 4ํㅡㄹición. México: Chicago Ediciones. 


\section{Omaira Andreina Márquez Ramírez \\ e-mail: omairamarquez89@gmail.com}

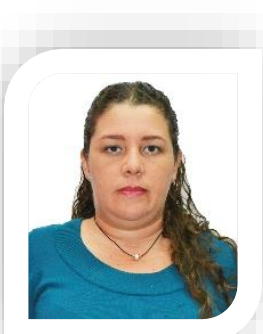

Natural de Barinas, Estado Barinas, Venezuela.

Profesora en Educación mención Educación Integral egresada de la Universidad Nacional Experimental de los Ilanos Occidentales Ezequiel Zamora (UNELLEZ). Actualmente se desempeña como profesora de aula en el N.E.R. 315 de la Parroquia San Silvestre en la Escuela Costa de Morrocoy Abajo.

Ha participado en Talleres como: Equipos de Trabajos Eficientes, Cursos de Operador de Micro bajo Ambiente Windows Milenium, curso de Primeros Auxilios, Conferencia Inteligencia Emocional, Certificado: Prácticas Profesionales III, como Excelente Compañera, su Puntualidad y Solidaridad durante el periodo de Pasantías en la Escuela Básica Bolivariana El Arenal. Núcleo Escolar Rural N.E.R. 147. Barinas Estado Barinas.

El contenido de este manuscrito se difunde bajo una Licencia de Creative Commons Reconocimiento- 